\title{
http://dx.doi.org/10.21611/qirt.1994.009 \\ Accuracy analysis of measuring thermal imaging systems
}

by CHRZANOWSKI K. and JANKIEWICZ Z.

Military University of Technology, Institute of Optoelectronics, 01-489 Warsaw, Poland

\section{Abstract}

A theory of the influence of measurement conditions and system parameters on the accuracy of remote temperature measurements with thermal imaging systems has been developed. An analysis of the influence of disturbances (such as: incorrectly assumed emissivity, radiation reflected by the object, radiation emitted by the optics of the system, limited transmittance of atmosphere and limited temperature resolution of the system) on the accuracy of temperature measurement has been made. The results have shown that the accuracy clearly differs in $3-5 \mu \mathrm{m}$ and $8-12 \mu \mathrm{m}$ spectral band under typical measurement conditions.

\section{Introduction}

Majority of thermal imaging systems (TISs) have been manufactured for military applications to enable observation, on the battlefield in darkness and under difficult atmospheric conditions, by producing the relative temperature distribution of the looked terrestrial scenery. The other, smaller group of TISs, has been developed for civilian applications in industry and science, to accurately measure temperature distributions on the surface of the tested objects. Therefore, according to application, the TISs can be divided into two groups: the observation (military) systems and the measuring (civilian) ones. Several studies [1-4] have been conducted to ovaluate the performance of observation systems and to create the theory of these systems in different conditions. The set of requirements of the observation thermal imaging systems is different from the set of the measuring ones. The primary factor that has to be considered for observation systems, is the signal to noise relationship at the output of the system, or the range of detection, recognition and identification of the object being observed. Instead, the primary factor that should be considered for measuring systems is the accuracy of determination of temperature distribution on the surface of the tested object. Because of the different set of requirements, the results of the studies [1-4] are not useful for the case of measuring TISs.

The problem of accuracy of measuring thermal imaging systems has been indirectly faced in several studies [5-7] which provide an analysis of them. However, the results have not yet explained the mechanism of the influence of the measurement conditions and system parameters on the accuracy of these systems. Recently, one of the manufactures of measuring thermal imaging systems has presented the results of a study [8]. This study is an overview on factors that affect and disturb the accuracy of remote temperature measurement and on how these factors differ between $3-5 \mu \mathrm{m}$ and $8-12 \mu \mathrm{m}$ thermal imaging systems. However, a theory explaining why the accuracy differs has not been presented so far.

In this paper a theory of the influence of the measurement conditions and system parameters on the accuracy of temperature measurement has been developed. The theory can be generally summarized with two relevant formulas. The first one describes the influence of the disturbances of the signal incident into the IR detector on the error of its measurement. The second one describes the relationship between the error of the signal measurement and the error of the temperature measurement. On the basis of the developed formulas, an analysis of the influence of the disturbances of the signal (due to: incorrectly assumed omissivity, radiation reflected by the object, radiation emitted by the atmosphere, radiation emitted by the optics of the system, limited transmittance of the atmosphere and limited 
http://dx.doi.org/10.21611/qirt.1994.009

temperature resolution of the system on the accuracy of temperature measurement) has been made.

\section{General theory}

To develop the present investigation several assumptions for the TIS are made in order to ease the problem. First, the case of a thermal imaging system, without an intemal temperature reference source, that is calibrated using an extemal one is considered. For such a case, imaging optics, scanner and filters can be treated as a single component: the optics of spectral transmittance $\tau_{0}(\lambda)$. Differences with the case of intemal reference source are discussed in detail in [6]. Second, the TIS is calibrated under laboratory conditions using a blackbody as a reference source. The distance between the blackbody and the system is short and it is assumed that, under such conditions, the influence of atmosphere and reflected background radiation is negligible. Third, two types of TIS are being compared: the short-wave, of spectral band $3-5 \mu \mathrm{m}$, and the longwave, of spectral band $8-12 \mu \mathrm{m}$. Identical optics, electronics, geometry, temperature and spatial resolutions are assumed. Fourth, the system works under indoor conditions.

If the above assumptions are fulfilled, the IR detector receives only the radiation emitted by the reference source and the radiation emitted by the optics within the detector spectral band. The value of the measured signal can be written, as

$$
S_{L}\left(T_{o b}, \varepsilon_{L}\right)=A\left[\varepsilon_{L} \int_{\lambda}^{\lambda} M\left(T_{o b}, \lambda\right) \tau_{o}(\lambda) s(\lambda) d \lambda+\int_{\lambda_{1}}^{h} M\left(T_{o p t}, \lambda\right)\left[1-\tau_{o}(\lambda)\right] s(\lambda) d \lambda\right]
$$

where $T_{o b}$ is the temperature of the reference source simulating the object, $T_{o p t}$ is the temperature of the optics, $s(\lambda)$ is the detector relative spectral detectivity function, $\tau_{0}(\lambda)$ is the transmittance of the system optics, $k$ is the constant of the signal transformation by the optics and the detector, $\lambda_{1}$ and $\lambda_{2}$ are the limits of the detector spectral band, and $M(T, \lambda)$ is the spectral emittance at the temperature $T$ and wavelength $\lambda$. Measuring TISs are usually calibrated using blackbodies as reference sources. The emissivity of real objects is always lower than emissivity of blackbodies. Therefore, to simulate the case of real objects (approximately grey bodies), the emissivity of the simulated object $\varepsilon_{L}$ has been added to equation (1).

The dependence of the signal $S_{L}\left(T_{o b}, \varepsilon_{L}\right)$ on the temperature $T_{o b}$ and the emissivity $\varepsilon_{L}$ of the simulated object, measured during the calibration process, is something like a standard during real measurements. It is generally assumed that a real object, under real conditions of temperature $T_{o b}$ and emissivity $\varepsilon_{L}$, gives the same signal as a reference source having the same temperature and emissivity during the calibration process. If this assumption is fulfilled and the TIS does not give any measurement errors, the temperature measurement error is null. However, the TISs always give measurement errors and, what is more important, the above assumption is rather rarely fulfilled. The signal measured under real work conditions usually differs from the one measured under laboratory conditions and the temperature of the tested object is determined with an error, even if the error due to the system is negligible. Let us treat all the sources, which produce differences between the signal measured under the laboratory and the real work conditions, as disturbances.

3. The influence of the disturbances of the measured signal on the accuracy of temperature measurement .

It is clear that disturbances of the signal measured by the system cause measurement errors of that signal and, consequently, cause temperature measurement errors. However, it is interesting how strongly the disturbances influence temperature measurement accuracy. Let 
http://dx.doi.org/10.21611/qirt.1994.009

us find the relationship between the signal measurement errors and temperature measurement errors. To find that relationship we have to carry out a few mathematical operations.

If we differentiate formula (1) and multiply it by the quantity $T_{o b} d T_{o b} / S_{L} T_{o b}$ we obtain

$$
\frac{d S_{L}}{S_{L}}\left(T_{o b}, \varepsilon_{L}\right)=D R F\left(T_{o b}, \varepsilon_{L}\right) \times \frac{d T_{o b}}{T_{o b}},
$$

where

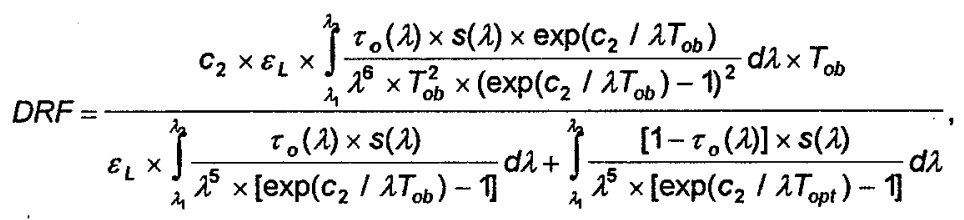

being $c_{1}, c_{2}$ the Planck constants.

For the case of small changes of the signal $S_{L}$, we could write the relationship between the signal measurement errors and the temperature measurement errors as

$$
\frac{\Delta T}{T_{o b}}=\frac{1}{D R F} \times \frac{\Delta S}{S_{L}}
$$

Equation (4) represents the relationship between the error of signal measurement caused by the disturbances and the error of temperature measurement. The new function $\operatorname{DRF}\left(T_{o b}, \varepsilon L, T_{\text {opt }}\right)$ can be called disturbance resistance function DRF because it represents the system resistance to the disturbances of the measured signal. The DRF gives us the information on how many times the error of temperature measurement is smaller than the error of signal measurement. It can be seen that the disturbance resistance function clearly prefers the short-wave TISs (figure 1). The values of the DRF are always higher for the short-wave TISs than for the longwave ones. This means that the same levels of disturbances of the measured signal causes lower errors of temperature measurement for the case of short-wave TISs. The rapid drop of the disturbance resistance functions at lower temperatures is the effect of the influence of the optics radiation. This radiation is negligible in the higher temperature range, but is important in the lower one, particularly for the case of low emissivity objects .

\section{Errors of the signal measurement due to disturbances.}

The results of the calculations from the previous section clearly show that the short-wave thermal imaging systems are more resistant to the disturbances of the signal received by the detector than the longwave ones. However, accuracy of the temperature measurement depends not only on the TIS resistance to the signal disturbances but also on the level of the disturbances. The disturbance resistance function DRF can be used as a useful figure of merit for comparison of thermal imaging systems, but the most important figure of merit for comparison of these systems is their accuracy of the temperature measurement.

Radiation incident onto IR detector under real work conditions consists of five components: the radiation emitted by the object, the radiation reflected by the object, the radiation emitted by the atmosphere, the radiation of sun (or other sources of strong radiation scattered in the atmosphere) and the radiation emitted by the optics. The first component depends on two parameters of the object: its temperature $T_{o b}$ and emissivity $\varepsilon_{R}$. The second component is generally the reflected radiation of object environment (the radiation of the sun, sky, the neighbouring objects and atmosphere) and depends on many environmental parameters. The Ihird component depends on the temperature of atmosphere, its spectral transmittance function $\tau_{a}(\lambda)$ and the distance $R$ between the object and the system. The fourth component depends, as the previous one, on the distance $R$, but also on many parameters of the 
http://dx.doi.org/10.21611/qirt.1994.009

atmosphere and the environment. The fifth component depends on the temperature of the optics $T_{\text {opt }}$ and its spectral transmittance function $\tau_{0}(\lambda)$.

The relative, apparent error of the signal measurement is expressed by the relationship between the difference of the signal measured under real work conditions $S_{R}$ and the value of the signal under laboratory ones $S_{L}$. We have

where

$$
\frac{\Delta S}{S_{L}}=\frac{S_{R}-S_{L}}{S_{L}}=A+B+C+D,
$$

$$
\begin{aligned}
& A=\frac{\int_{1}^{\lambda_{1}} \frac{\varepsilon_{R}(\lambda) \times \tau_{a}(\lambda) \times \tau_{o}(\lambda) \times s(\lambda)}{\lambda^{5} \times\left[\exp \left(c_{2} / \lambda T_{o b}\right)-1\right]} d \lambda-\varepsilon_{L} \times \int_{\lambda_{1}}^{\lambda_{1}} \frac{\tau_{o}(\lambda) \times s(\lambda)}{\lambda^{5} \times\left[\exp \left(c_{2} / \lambda T_{o b}\right)-1\right]} d \lambda}{\varepsilon_{L} \times \int_{\lambda_{1}}^{\lambda^{5} \times\left[\exp \left(c_{2} / \lambda T_{o b}\right)-1\right]} d \lambda+\int_{\lambda_{1}}^{\lambda_{0}(\lambda) \times s(\lambda)} \frac{\left[1-\tau_{o}(\lambda)\right] \times s(\lambda)}{\lambda^{5} \times\left[\exp \left(c_{2} / \lambda T_{o p t}\right)-1\right]} d \lambda},
\end{aligned}
$$

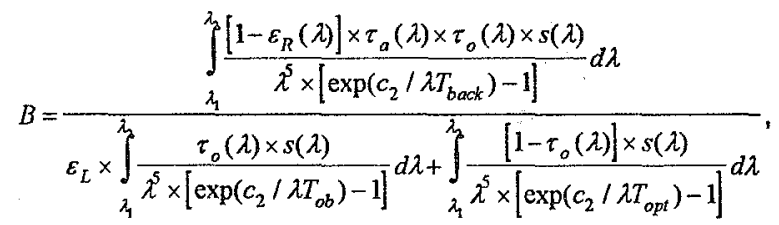

$$
\begin{aligned}
& C=\frac{\left.\int_{\lambda^{2}}^{\lambda^{5} \times\left[\exp \left(c_{2} / \lambda T_{o p t}^{r}\right)-1\right]} d \lambda\right]-\int_{\lambda_{1}}^{\lambda_{1}} \frac{\left[1-\tau_{o}(\lambda)\right] \times s(\lambda)}{\lambda^{5} \times\left[\exp \left(c_{2} / \lambda T_{o p t}\right)-1\right]} d \lambda}{\lambda_{1}^{\lambda_{1}} \frac{\tau_{o}(\lambda) \times s(\lambda)}{\lambda^{5}\left[\exp \left(c_{2} / \lambda T_{o b}\right)-1\right]} d \lambda+\int_{\lambda_{1}}^{\lambda_{1}} \frac{\left[1-\tau_{o}(\lambda)\right] \times s(\lambda)}{\lambda^{5} \times\left[\exp \left(c_{2} / \lambda T_{o p t}\right)-1\right]} d \lambda},
\end{aligned}
$$

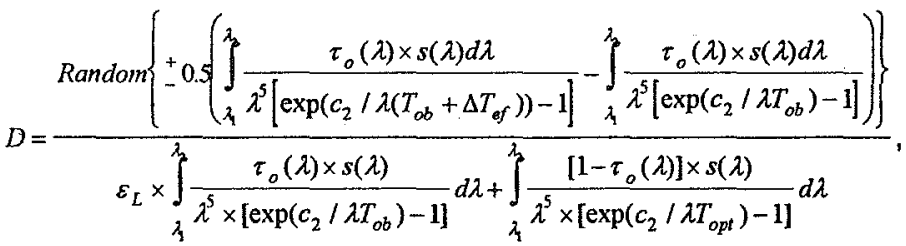

where $\varepsilon_{R}$ is the real emissivity of the tested object, $T_{\text {back }}$ is the temperature of the background, $T_{\text {opt }}^{r}$ is the temperature of the optics under real work conditions, $\Delta T_{\text {er }}$ is the system effective temperature resolution, function Random represents uniformly distributed pseudo random amplitude values equal to the uncertainties of the signal measurement due to the limited temperature resolution.

\section{Errors of the temperature measurement}

The components $A, B, C, D$ give informations on the error of the signal measurement due to the disturbances: the component $A$ on the effect of improperly assumed emissivity (when $\tau_{a}(\lambda)$ $=1$ ) and on the effect of limited transmittance of the atmosphere (when emissivity $\left.\varepsilon_{R}(\lambda)=\varepsilon_{L}\right)$; the component $B$ on the influence of the reflected radiation (when $\tau_{a}(\lambda)=1$ ) and on the effect of limited transmittance (when $T_{\text {back }}$ is low); the component $C$ on the effect of changes of the optics radiation; the component $D$ on the effect of uncertainties which arise during the signal measurement, due to the limited temperature resolution of the system. The total error of the 
signal measurement is the sum of all these components. The error of the temperature measurement is a product of the error of the signal measurement and the disturbance resistance function of the system according to formula (4). The errors of temperature measurement (due to the: improperly assumed emissivity, reflected radiation, limited transmittance of the atmosphere, optics radiation and limited temperature resolution of the system for simulated conditions) are presented in figures 2-6. The calculations have been made for typical detectors and optics optimised for the 3-5 $\mu \mathrm{m}$ and $8-12 \mu \mathrm{m}$ "atmospheric window" and the following simulated conditions: figure 2 - relative error of the emissivity determination equal to $5 \%$; figure 3 - background temperature $T_{\text {back }}=303 K$; figure 4 atmosphere temperature $T_{\text {atm }}=297 \mathrm{~K}$, object temperature $T_{o b}=500 \mathrm{~K}$, atmosphere relative humidity equal to $50 \%$; figure 5 - optics temperature under real work conditions equal to $303 K$ and under laboratory conditions equal to 297K; figure 6 - temperature resolution for both systems equal to $0.1 \mathrm{~K}$ at temperature $300 \mathrm{~K}$.

\section{Conclusions.}

Accuracy of temperature measurement is one of the most important factor when comparing thermal imaging systems. The results presented above clearly show that the errors of temperature measurements due to analysed disturbances are generally smaller for the shortwave $3-5 \mu \mathrm{m}$ systems. The longwave systems behave exceptionally poorly when compared to the short-wave ones for the case of low emissivity objects. The only exception are the errors due to the influence of limited transmittance of the atmosphere, that are smaller for the longwave $8-12 \mu \mathrm{m}$ systems. Yet, these errors are small for short paths, even for the short-wave systems. Therefore, one can formulate a general rule that the short-wave systems behave better than the longwave ones. However, we have to remember that the study has been limited to systems working under indoor conditions. Consequently, the conclusion, formulated earlier, is valid only for this group of measuring systems.

Even under indoor conditions there are two exceptions to the rule. First, in general, the short-wave systems are more accurate in the temperature range from $270 \mathrm{~K}$ to $900 \mathrm{~K}$. There is, however, the small part of this range where the longwave systems perform better tomperatures below $300 \mathrm{~K}$. In fact there are some applications where it is necessany to measure temperatures below this value, although hotter objects are usually measured in typical industrial practice. Second, the influence of the atmosphere has been analysed only for one example of the transmission of typical atmosphere. However, dust, smoke and gases are sometimes met under industrial conditions. Their presence usually affects more the atmospheric transmission in $3-5 \mu \mathrm{m}$ band and may clearly worsen performance of the shortwave systems.

The values of the relative spectral detectivity of the IR detectors $s(\lambda)$, the optics If ansmittance $\tau_{0}(\lambda)$, the atmosphere transmittance $\tau_{a}(\lambda)$, the temperature of the background $T_{\text {back }}$, the temperature of the atmosphere $T_{\text {atm }}$, and the difference of the optics temperature under laboratory and real work conditions used in the calculations can be regarded as typical for a real measurement process. The errors of temperature measurement have been calculated for objects at temperatures within the temperature range of most thermal imaging systems. We can say that the assumptions should not reduce the accuracy of the simulated systems. Calculated errors of temperature measurement are quite significant, particularly for low emissivity objects in the lower temperature range. The errors typically decrease when the object temperature rises. But, there is an exception - the errors due to improperly assumed emissivity. So, the errors of temperature measurement with thermal imaging systems cannot bo treated as negligible in a whole typical temperature range and they are many times higher than for contact temperature measurement devices. However, one should notice that, with the axception of the errors due to limited temperature resolution, the errors can be treated as constant, independent on time. This fact enables us to improve accuracy of temperature measurement when using thermal imaging systems by correcting these errors and modern aystems [8] do it. The developed theory of the influence of the measurement conditions and the system parameters on the accuracy of temperature measurement has been used in this 
http://dx.doi.org/10.21611/qirt.1994.009

paper to calculate the errors of temperature measurement under simulated conditions. It can be also used to develop software models in oder to correct them.

\section{REFERENCES}

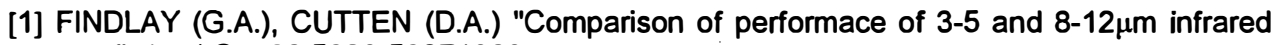
systems", Appl.Opt.28,5029-50371989.

[2] JOHNSON (R.B.)"Relative merits of the 3-5 $\mu \mathrm{m}$ and $8-12 \mu \mathrm{m}$ spectral bands," SPIE,917,102107, 1988.

[3] JAEGER (T.) NORDBRYHN (A.) STOKSETH (P.A.)," Detection of low contrast targets at 5 $\mu \mathrm{m}$ and 10 $\mu \mathrm{m}$ :a comparison", Appl. Opt. 11,1833-1835, 1972.

[4] LONGSHORE (R.), RAIMONDI (P.), LUMPKIN (M.),"Selection of detector peak wavelength for optimum infrared systems performance", Infrared Phys.,16,639-647, 1976.

[5] ABEL (J.A.),"Radiometrics accuracy of forward looking infrared systems", Opt. Eng.16,241248, 1977.

[6] BRAIM (S.P.),"Technique for the analysis of data from an imaging infrared radiometer", Infrared Phys. 28, 255-261, 1988.

[7] FRAEDRICH (D.S.), "Method in calibration an error analysis for infrared imaging radiometer", Opt. Eng. 30, 1764-1768, 1988.

[8] HAMRELIUS (T.), "Accurate temperature measurement in thermography", Quantitative Infrared Thermography QIRT 92, EUROTHERM Seminar 27, Chatenay-Malabry, France, 1992. 
http://dx.doi.org/10.21611/qirt.1994.009

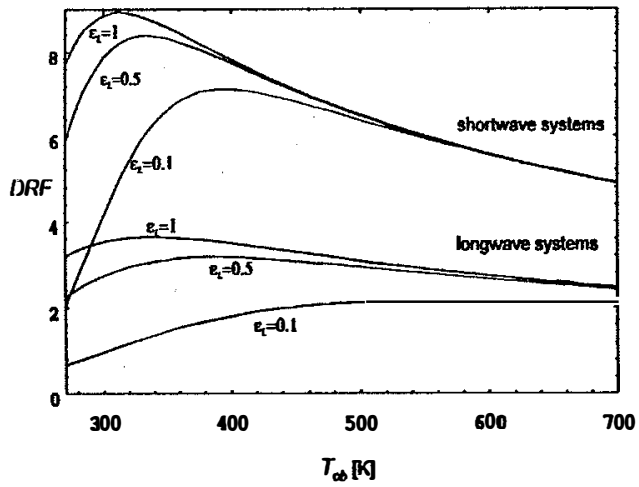

Flg.1- The disturbance resistance functions DRF

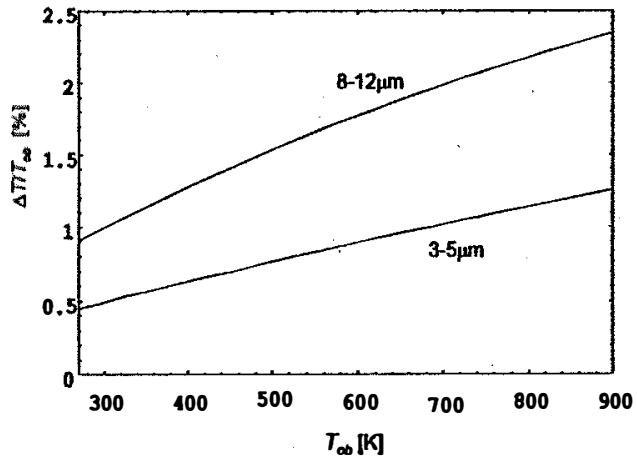

Fig. 2 - Relative error of temperature measurement due to improperty assumed emissivity.

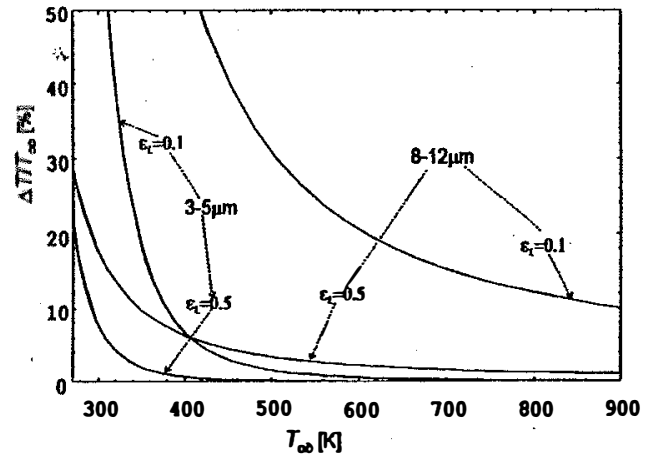

Fig.3 - The relative error of temperature measurement due to the refiected radiation.

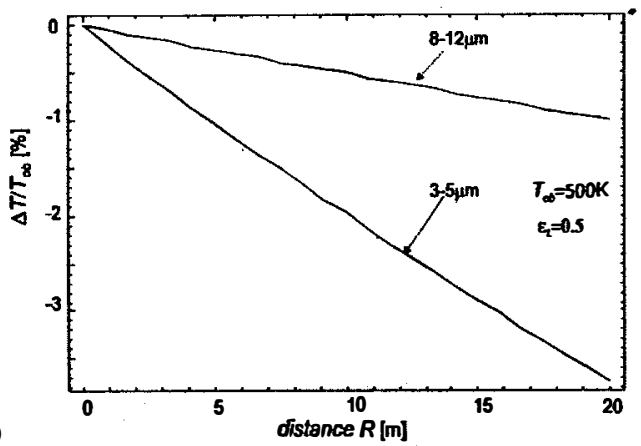

Fig.4 - The errors of the measured signal due to the limited transmittance of the atmosphere.

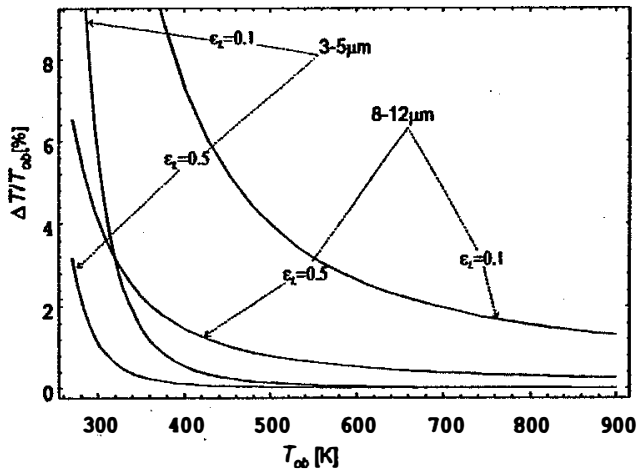

Fig.5 - The errors of the temperature measurement due to the radiation emitted by the optics.

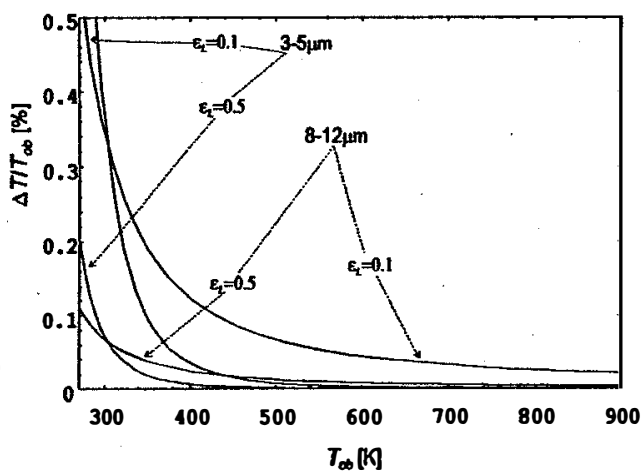

Fig.6 - The errors of the temperature measurement due to the limited temperature resolution of the system. 